\title{
Gumi Bao decoction regulates bone metabolism-related mRNA expression in glucocorticoid-induced osteoporosis in rats
}

\author{
Huanhuan Feng ${ }^{1,2} \wedge$, Zhengfeng Gong ${ }^{3}$, Guoqiang Liang ${ }^{3,4}$, Xiaofeng Shen ${ }^{3}$, Jian Cheng ${ }^{1,5}$, Fangdong Su ${ }^{2}$, \\ Lizhong Yang ${ }^{2}$, Hua Liu ${ }^{2}$ \\ ${ }^{1}$ Department of First Clinical Medical Institute, Nanjing University of Chinese Medicine, Nanjing, China; ${ }^{2}$ Department of Orthopedics, Taicang \\ TCM Hospital Affiliated to Nanjing University of Chinese Medicine, Taicang, China; ${ }^{3}$ Department of Orthopedics, Suzhou TCM Hospital \\ Affiliated to Nanjing University of Chinese Medicine, Suzhou, China; ${ }^{4}$ Suzhou Academy of Wumen Chinese Medicine, Suzhou, China; ${ }^{5}$ Department \\ of Orthopedics, Xuzhou Central Hospital, Xuzhou, China \\ Contributions: (I) Conception and design: H Feng, Z Gong; (II) Administrative support: Z Gong; (III) Provision of study materials or patients: G \\ Liang, X Shen; (IV) Collection and assembly of data: H Feng, G Liang, J Cheng, F Su; (V) Data analysis and interpretation: H Feng, G Liang, H \\ Liu, L Yang; (VI) Manuscript writing: All authors; (VII) Final approval of manuscript: All authors. \\ Correspondence to: Huanhuan Feng. Department of Orthopedics, Taicang TCM Hospital Affiliated to Nanjing University of Chinese Medicine, \\ Taicang, China. Email: fhh420@163.com.
}

Background: Gumi Bao decoction is effective for the treatment of osteoporosis, but the theoretical and scientific basis is unknown. This study aimed to observe the effect of Gumi Bao decoction on Dickkopf-related protein 1 (DKK1) mRNA, runt-related transcription factor 2 (Runx2) mRNA, and cathepsin K (CTSK) mRNA in glucocorticoid-induced osteoporosis (GIOP) in rats and to investigate the underlying mechanism.

Methods: Sixty Sprague-Dawley (SD) rats were weighed and randomly divided into six groups: the normal control group (NC group), the methylprednisolone group (Met group), the Fosamax group, the low-dose Gumi Bao Decoction group (GBDL group), the medium-dose Gumi Bao Decoction group (GBDM group), and the high-dose Gumi Bao Decoction group (GBDH group). The basic physiological conditions of the rats and the bone morphology of the fourth lumbar vertebra and the left femur of three rats in each group were observed, and the mRNA expressions of bone tissue-related genes were detected.

Results: After administration, DKK1 mRNA expression was significantly up-regulated in the Met group compared to the NC group $(\mathrm{P}<0.001)$. Meanwhile, DKK1 mRNA was significantly down-regulated in the Fosamax group compared with the Met group $(\mathrm{P}<0.001)$. There was a significant difference in the down-regulation of DKK1 mRNA between the GBDM and GBDH groups $(\mathrm{P}<0.001)$. Runx2 mRNA was considerably down-regulated in the Met group compared with the $\mathrm{NC}$ group $(\mathrm{P}<0.001)$. Runx2 mRNA was up-regulated in the GBDM group, and the GBDH group was significantly different compared to the Met group $(\mathrm{P}<0.001)$. CTSK mRNA was significantly up-regulated in the Met group compared to the NC group $(\mathrm{P}<0.001)$. Compared with the Met group, CTSK mRNA expression was significantly down-regulated in the Fosamax group $(\mathrm{P}<0.001)$, as well as in the GBDL, GBDM, and GBDH groups $(\mathrm{P}<0.001)$. At 200x and $400 \times$, there were significantly fewer osteoblasts and osteoclasts in the Met group than in the Fosamax, GBDM, and GBDH groups.

Conclusions: The imbalance of bone homeostasis of GIOP is caused by an increase in bone resorption and decreased osteogenesis. Gumi Bao could regulate bone metabolism through the action of DKK1 via the Wnt/ $\beta$-catenin signaling pathway, up-regulating Runx2 mRNA and down-regulating CTSK mRNA.

Keywords: Gumi Bao decoction; glucocorticoid; osteoporosis; glucocorticoid-induced osteoporosis (GIOP)

Submitted Jun 03, 2020. Accepted for publication Aug 21, 2020.

doi: 10.21037/apm-20-1340

View this article at: http://dx.doi.org/10.21037/apm-20-1340

$\wedge$ ORCID: 0000-0001-7856-3485. 


\section{Introduction}

Glucocorticoid-induced osteoporosis (GIOP) is a common side effect of glucocorticoid (GC) therapy and is the most frequent form of secondary osteoporosis (1). Longterm exogenous GC use eventually destroys microscopic bone structure and bone mass, resulting in secondary osteoporosis (2-4). Existing clinical data show that the risk of bone fracture from GC therapy increases along with the length of use and dosage. Heavy users of GCs carry a greater risk of sustaining bone fractures compared with short-term users (5-8). Fracture is a serious complication of osteoporosis. The incidence of fractures among adult patients aged between 20 and 45 years old who receive long-term GC therapy is as high as $30-50 \%(9,10)$. This places a considerable financial and social burden on patients and their families. Therefore, it is crucial to prevent GIOP by regulating bone metabolism.

The underlying mechanism of GIOP is believed to involve the decrease of osteoblast activity and the enhancement of osteoclast activity, leading to decreased bone formation and increased bone destruction (11-13). Dickkopf-related protein 1 (DKK1), runt-related transcription factor 2 (Runx2), and cathepsin K (CTSK) are involved in the action mechanism of Chinese herbal medicine compounds. DKK1 was only expressed in osteoblasts and osteocytes. It is an inhibitor of the Wnt/ $\beta$-catenin signaling pathway and serves as an important target for the prevention and treatment of GIOP $(14,15)$. It exerts an inhibitive effect on Wnt signaling pathway transduction by competitively binding to $\mathrm{Wnt}$ protein Frizzled-LRP5/6 complex receptors to reduce the binding rate of Wnt ligands (16). Runx2 is an osteogenic marker. Runx 2 mRNA is a target of the bone morphogenetic protein pathway and is an essential factor in the regulation of osteoblast differentiation (17). Meanwhile, monocyte chemotactic protein-1 can induce the differentiation of monocytes into cathepsin K-positive osteoclast precursors. Osteoclasts present CTSK mRNA, which causes bone destruction (18).

In traditional Chinese medicine, osteoporosis belongs to the category of "bone witherosis". Experiments have proved that traditional Chinese medicine compounds can inhibit bone absorption, promote bone formation, and effectively prevent GIOP. Gumi Bao decoction plays an active role in the prevention and treatment of osteoporosis; however, its prevention mechanism has yet to be fully elucidated. Therefore, this study aimed to investigate the effects of
Gumi Bao decoction on DKK1 mRNA, Runx2 mRNA, and CTSK mRNA in GIOP in rats and to investigate the theoretical and scientific basis.

We present the following article in accordance with the ARRVIE reporting checklist (available at http://dx.doi. org/10.21037/apm-20-1340).

\section{Methods}

\section{Laboratory animals and grouping}

Healthy female Sprague-Dawley (SD) rats (180-220 g) were supplied by JOINN Laboratories (Suzhou, China; license number: SCXK [su] 2017-0043). The rats were fed with special rat food for 1 week and housed indoors under the same light and humidity conditions at $22-26^{\circ} \mathrm{C}$. After 5 days of adaptive feeding, $60 \mathrm{SD}$ rats were weighed and divided equally according to the random number table method into the following six groups: the normal control group (NC group), the methylprednisolone group (Met group), the Fosamax group, the low-dose Gumi Bao decoction group (GBDL group), the medium-dose Gumi Bao decoction group (GBDM group), and the high-dose Gumi Bao decoction group (GBDH group). Experiments were performed under a project license (ID: 201901) granted by the Animal Care Welfare Committee of Taicang TCM Hospital Affiliated to Nanjing University of Chinese Medicine, in compliance with Chinese national guidelines for the care and use of animals.

\section{Experimental drugs}

Gumi Bao decoction consists of Milkvetch Root (20 g), Malaytea Scurfpea Fruit (10 g), Tuber Fleeceflower Root $(15 \mathrm{~g})$, Desertliving Cistanche Herb (10 g), Twotoothed Achyranthes Root (10 g), Acruginous Turmeric Rhizome (10 g), and Cuttlebone Sepium $(30 \mathrm{~g})$. All of the drugs were provided by Taicang TCM Hospital Affiliated to Nanjing University of Chinese Medicine.

Decoction method: The pure ingredients were soaked in water for 30 minutes and then decocted in a Chinese herbal medicine decoction machine for 40 minutes. This step was repeated twice. Then, two different concentrations of the decocting solution were pooled together, put into an electrothermal constant temperature water tank, and diluted to a concentration of $2.0 \mathrm{~g}$ of Gumi Bao decoction per $\mathrm{mL}$. The samples were sealed and stored in a refrigerator at $4{ }^{\circ} \mathrm{C}$. The validity of the decoction was set to 1 week, and it was 
prepared weekly.

\section{Main instruments and reagents}

The materials and instruments used included the DHG9240 incubator (Shanghai Yiheng Scientific Instrument Co., Ltd, Shanghai China), Infinite F50 enzyme marker (Tecan, Switzerland); RT-3500 board washing machine (Shenzhen Leidu, Shenzhen, China), Sorvall ST40 Centrifuge (Thermo Fisher Scientific, USA), a reverse transcription kit (Fermenta Biotech Ltd, USA); Trizol Kit (Invitrogen, USA), ABI7300 Real-time detector (ABI, USA), TG-16M cryogenic centrifuge (Shanghai Luxiangyi Centrifuge Instrument Co., Ltd, Shanghai, China), K30 vortex oscillator (Qingpu Luxi Instrument Factory, Shanghai, China), PRO200 electric homogenizer (FLUKO, Shanghai, China), a microscope (Olympus CX41, Japan), a constant temperature oven (Shanghai Hengyi Scientific Instrument Co., Ltd, Shanghai, China), a paraffin slicing machine (Leica SQ2125, Hubei, China), a tablet spreading machine (Leica PPTHK-21B, Hubei, China), a water bath (Leica HI1210, Hubei, China), and a digital camera (NIKON D5100, Japan).

\section{Establishment of experimental animal of GIOP and treatment}

Hulley's method (19) was used to establish a GIOP rat model. The Met group was subcutaneously injected with methylprednisolone $(5.0 \mathrm{mg} / \mathrm{kg}$, once a day). After the model had been successfully established, the rats in the Fosamax group were given Fosamax ( $2 \mathrm{mg} / \mathrm{kg}$, once a day) via intragastric administration. The rats in the GBDL $(5.0 \mathrm{~g} / \mathrm{kg})$, GBDM $(10.0 \mathrm{~g} / \mathrm{kg})$, and GBDH $(20.0 \mathrm{~g} / \mathrm{kg})$ groups were intragastrically administered Gumi Bao decoction once a day. The rats in the NC group received a daily subcutaneous injection of normal saline $(0.75 \mathrm{~mL} / \mathrm{kg})$ once a day. Each group was given intervention for 6 weeks, during which time the rats were weighed weekly and the drug dose was adjusted according to the body mass.

\section{Sample collection and detection}

\section{General observations}

During the experiment, the basic physiological conditions of the rats, such as food and water intake, movement, and skin color, were closely observed. Changes in the body mass of the rats were measured with a balance during the experiment.

\section{Detection of bone tissue-related mRNA}

Rats in each group were euthanized by cervical dislocation, and the second lumbar vertebra was collected. Total RNA was extracted with a Trizol kit, after which the mRNA expressions of DKK1, Runx2, and CTSK were detected by reverse transcription and amplification by real-time polymerase chain reaction (PCR) (Figures 1-3). The data were analyzed with ABI Prism 7300 SDS Software. Primers were purchased from Shanghai Banyi Biological Co., Ltd (Shanghai, China) (Table 1).

\section{Bone morphology}

The fourth lumbar vertebra and the left femur of three rats from each group were collected. The samples were sequentially decalcificated, embedded, fixed, sectioned, and stained with hematoxylin and eosin (H\&E).

\section{Statistical analysis}

Data analysis was performed with SPSS 22.0 (IBM, USA). The data were expressed as the mean \pm standard $(\bar{x} \pm S)$. The measurement data were analyzed by oneway analysis of variance (ANOVA) when the data satisfied the assumption of homogeneity of variance, or at least significant difference test (LSD-t) was performed for pairwise comparisons. $\mathrm{P}<0.05$ was considered to represent statistically significant difference.

\section{Results}

\section{General observations of the rats}

During the intervention, the rats in the Met group gradually became lethargic, their fur became sparse and lackluster, and they had lower food intake and slow body mass growth. In contrast, the rats in the $\mathrm{NC}$ group showed no signs of lethargy and had shiny fur. In the first 3 weeks of intervention, the rats in the Fosamax, GBDM, and GBDH groups exhibited sparse and lackluster fur, lethargy, and less food intake, but their conditions gradually improved in the second 3 weeks. No obvious improvement was observed in the GBDL group compared with the Met group.

During the experiment, 1 rat in the Met group died from infection and 1 rat in the GBDL group died from gavage. After the intervention, the weight of the rats in the GBDL group was significantly decreased compared to that of the $\mathrm{NC}$ group rats $(\mathrm{P}<0.001)$. Meanwhile, the weight of the rats in the Fosamax, GBDM, GBDH $(\mathrm{P}<0.01)$, and GBDL 


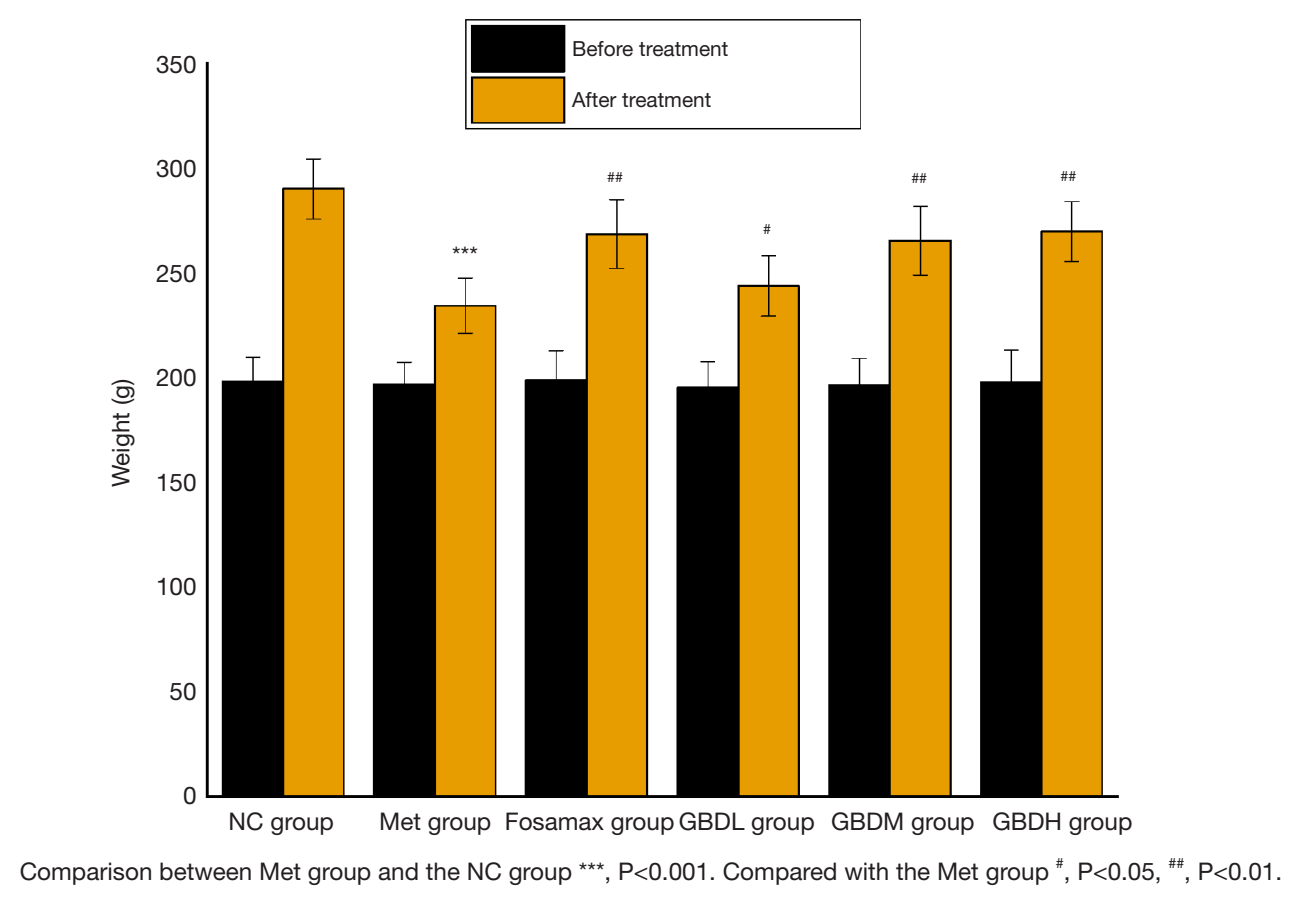

Figure 1 The weights of rats before and after treatment $(\bar{x} \pm s)$.

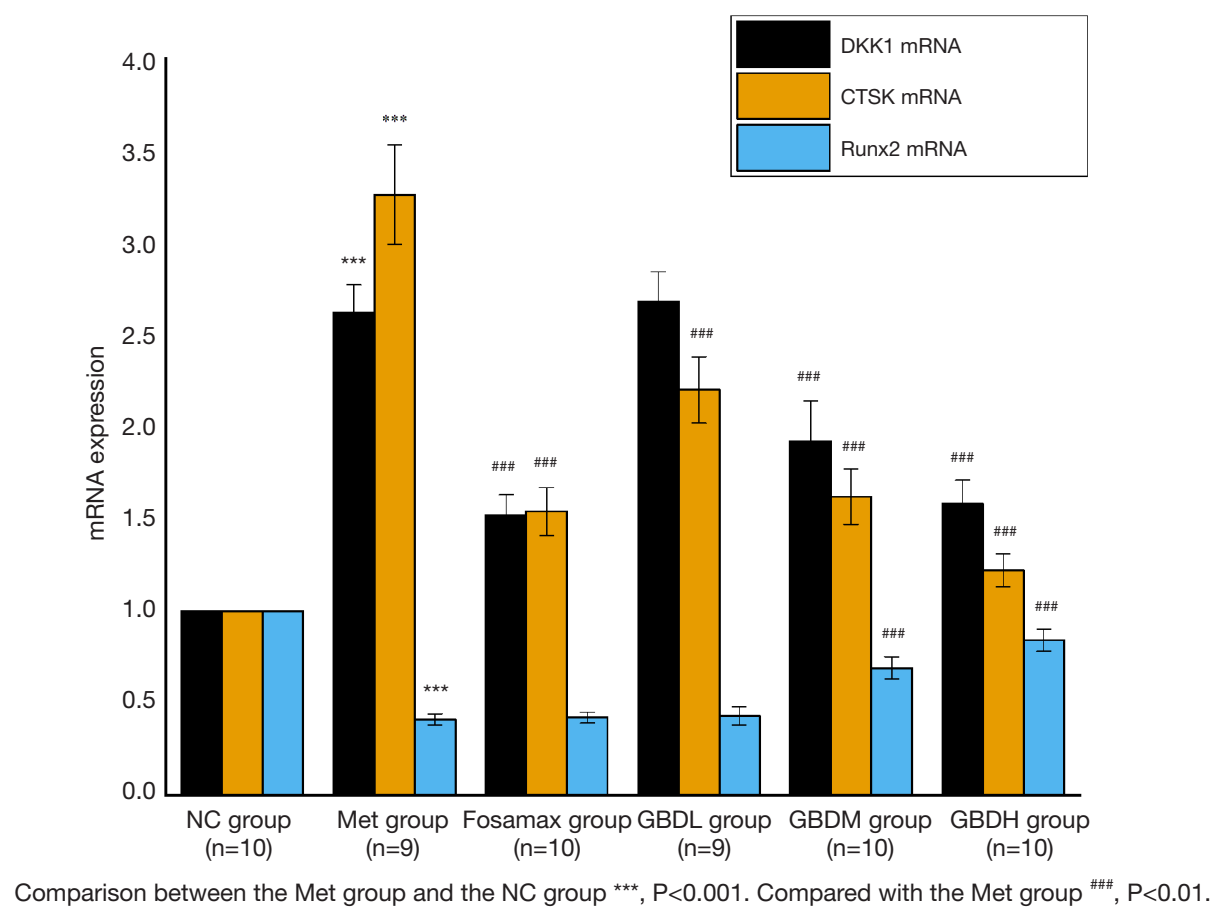

Figure 2 Determination of DKK1 mRNA, Runx2 mRNA, and CTSK mRNA expression in L2 bone tissues of rats after treatment $(\bar{x} \pm s)$. 

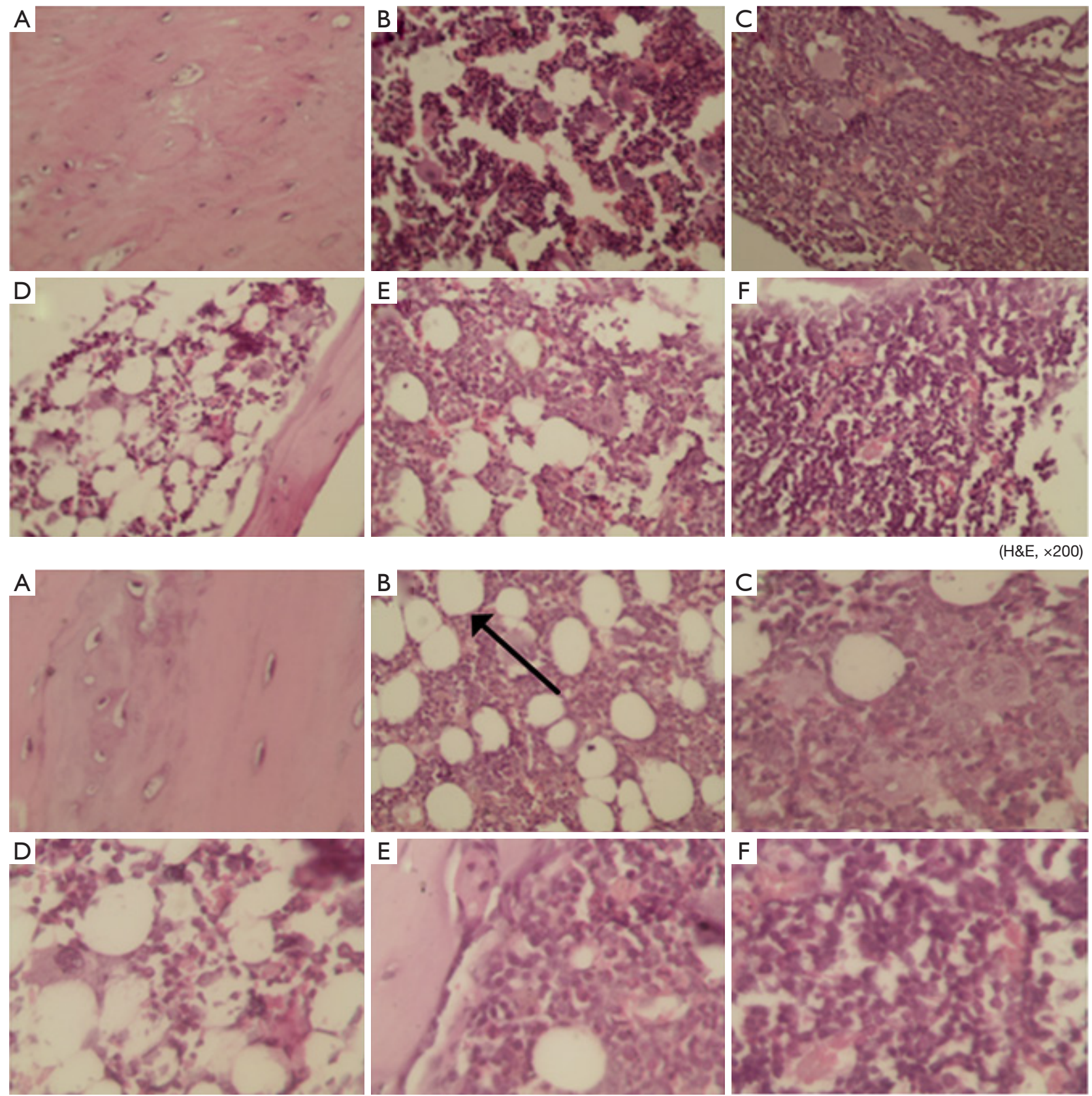

$(\mathrm{H} \& \mathrm{E}, \times 400)$

Figure 3 H\&E staining of rat femur tissues. (A) NC group; (B) Met group; (C) Fosamax group; (D) GBDL group; (E) GBDM group; (F) GBDH group. 
Table 1 Primer sequences

\begin{tabular}{ll}
\hline Gene & Primer (5'-3') \\
\hline DKK1 & F: 5'-CGGTTCTTGGTCGTGCTTTC-3' \\
& R: 5'-AAGGGTAGGGCTGGTAGTTG-3' \\
Runx2 & F: 5'-ACTTCGTCAGCGTCCTATC-3' \\
& R: 5'-CATCAGCGTCAACACCATC-3' \\
CTSK & F: 5'-CGACCGTGATAATGTGAACC-3' \\
& R: 5'-GCACTGTTGAGGAAGGAATG-3' \\
GAPDH & F: 5-'GGAGTCTACTGGCGTCTTCAC-3' \\
& R: 5'-ATGAGCCCTTCCACGATGC-3'
\end{tabular}

Methylprednisolone was purchased from Pfizer Manufacturing Belgium NV (Belgium). Fosamax was obtained from Hangzhou MSD Pharmaceutical Co., Ltd. (Hangzhou, China). Pentobarbital sodium was purchased from Shanghai Rongbai Biotechnology Co., Ltd. (Shanghai, China).

$(\mathrm{P}<0.05)$ groups was significantly increased compared with the Met group (Figure 1).

\section{Administration of DKK1, Runx2, and CTSK mRNA expression in $L 2$ bone tissues of rats}

After the intervention, there was a significant difference in the up-regulation of DKK1 mRNA expression in the Met group in contrast to the $\mathrm{NC}$ group $(\mathrm{P}<0.001)$. Compared with the Met group, there was a vast difference in the down-regulation of DKK1 mRNA in the Fosamax group $(\mathrm{P}<0.001)$. There was a significant difference in the downregulation of DKK1 in the GBDM group and GBDH group $(\mathrm{P}<0.001)$ and no difference in the GBDL group $(\mathrm{P}>0.05)$. The down-regulated expression of Runx2 in the Met group was significantly different from that in the $\mathrm{NC}$ group $(\mathrm{P}<0.001)$. There was no significant difference in Runx2 mRNA expression between the Met group and the Fosamax and GBDL groups $(\mathrm{P}>0.05)$. The expression of Runx2 mRNA was significantly up-regulated in the GBDM group compared with the GBDH group $(\mathrm{P}<0.001)$, while there was no significant difference between Runx2 expression in the GBDL group and the Met group $(\mathrm{P}>0.05)$. Vertebral osteoclast regulator CTSK mRNA was significantly up-regulated in the Met group compared with the NC group $(\mathrm{P}<0.001)$, and CTSK mRNA expression in the Fosamax, GBDL, GBDM, and GBDH groups was significantly down-regulated compared with the Met group $(\mathrm{P}<0.001)$ (Figure 2).

\section{Morphological changes in the femur and L4 in rats}

As shown in Figure 3, there were fewer osteoblasts and osteocytes in the trabecular bone of the Met group (B) than in that of the Fosamax group (C) and the GBDH group (F) under 200× H\&E staining. The bone tissue of each group of rats (B,C,D,E,F) other than the NC group (A) showed structural destruction of trabecular bone to varying degrees. Among the groups of $200 \times \mathrm{H} \& \mathrm{E}$ staining, the bone structure of the Met (B) and GBDL (D) groups was seriously damaged. The phenomenon of H\&E staining at $400 \times$ was similar to that at $200 x$. The images from the GBDH (F) and Fosamax (C) groups reveal the integrity of the structure of trabecular bone and show that no adipose tissue was formed. A predominance of adipose tissue (marked by black arrows) was found in the Met (B) and GBDL (D) groups, and a small amount of adipose tissue had formed in the Fosamax (C) and GBDM (E) groups.

Figure 4 shows H\&E staining (at 200x and 400x) of the rat L4 spinal sections. In contrast with the NC group (A), the trabecular bone was structurally intact and osteocytes and osteoblasts were abundant in the GBDM (E) and GBDH (F) groups. The structural destruction of trabecular bone was obvious in the GBDL group (D). A large amount of adipose tissue under 400× H\&E staining could be seen in GBDL group (D), but there was less in the GBDM group (E). In the GBDH (F) and Fosamax (C) groups, the bone structure was relatively intact, and there was less adipose tissue.

\section{Discussion}

In the theory of traditional Chinese medicine, longterm GC use can easily damage Yin fluid, and kidney Yin deficiency is insufficient for the metaplasia of kidney Yang. This causes deficiency of kidney Yang and kidney Yin, bone deficit, marrow reduction, and blood stasis, which ultimately leads to GIOP. During intervention with methylprednisolone, the rats in the Met group gradually displayed sparse and lackluster fur, lethargy, low food intake, and slow growth of body mass. Compared with the Met group, the weight of the rats in the Fosamax group and the GBDL, GBDM and GBDH groups was significantly increased. Gumi Bao decoction not only nourishes the essence of Yin and Yang to enrich the kidney, but also nourishes the source of acquired biochemistry to strengthen spleen qi. Traditional Chinese medicine for invigorating blood and removing blood stasis can also have the effect of tonifying the kidney without stagnation. 

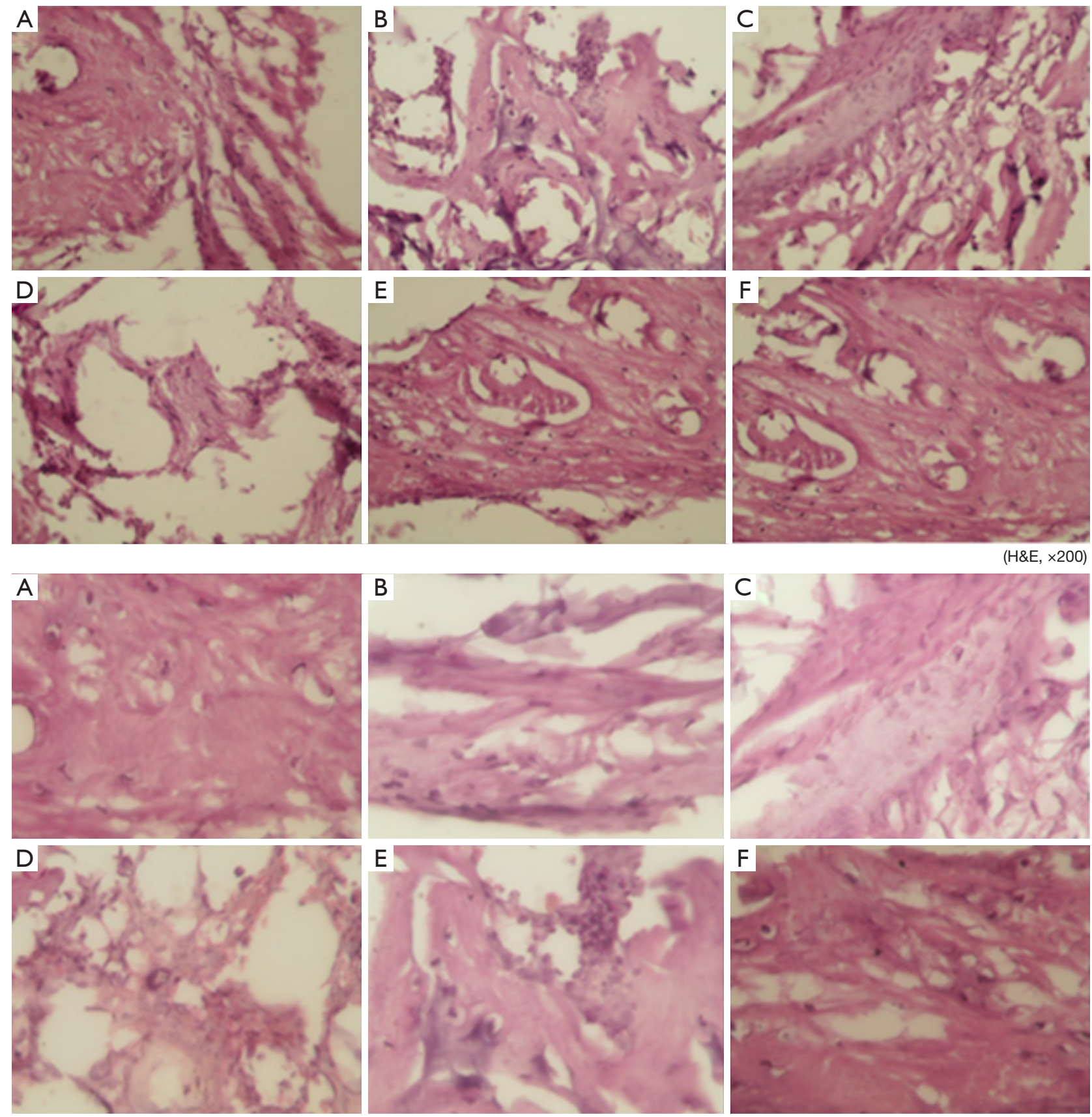

$(\mathrm{H} \& \mathrm{E}, \times 400)$

Figure 4 H\&E staining of rat L4 tissue. (A) NC group; (B) Met group; (C) Fosamax group; (D) GBDL group; (E) GBDM group; (F) GBDH group.

Dickkopf-related proteins are important inhibitors of the $\mathrm{Wnt} / \beta$-catenin signaling pathway, which regulates osteoblast differentiation and promotes osteogenesis. Studies have shown that GCs can induce the up-regulation of DKK1 expression to inhibit the $\mathrm{Wnt} / \beta$-catenin signaling pathway. The expression level of DKK1 directly affects the transduction of the downstream Wnt signaling pathway and plays a major role in the regulation of osteogenic differentiation. The expression level of DKK1 in bone tissue was inversely proportional to the bone mass of the rats, and 
the increase of serum DKK1 level was closely related to the destruction of human bone microstructures (20). Our study showed that after 6 weeks of intervention, osteoblasts and osteocytes in the vertebral body and femoral bone tissues of the rats in the Met group had sustained damaged, with upregulated expression of DKK1.

GCs could reduce the expression of the bone formationassociated gene Runx2 (21). Runx2 is a target of bone morphogenetic proteins and a key factor in the regulation of osteoblast differentiation. Runx2 is a transcription factor in the runt-related gene family, which regulates osteogenic differentiation by binding to specific DNA sequences and up-regulates the transcription of various mineral-related protein genes in pre-osteoblasts and chondrocytes. Runx2 promotes osteogenesis and inhibits adipogenesis in bone mesenchymal stem cells (BMSCs) (22). In this study, Runx2 was down-regulated in rats after GC intervention. Compared with the Met group, Runx2 mRNA was upregulated after intervention with Gumin Bao decoction, which suggests that Gumin Bao decoction can regulate DKK1 to promote the prevention and treatment of GIOP.

CTSK is an osteoporosis-targeted protease, and activated osteoclasts specifically express mature CTSK. CTSK accumulates in osteoclast lysosome vesicles and is released into the extracellular absorption lacunae in the acidic environment. CTSK can degrade type I collagen: type I collagen $\mathrm{N}$-terminal peptide can be degraded to produce $\mathrm{N}$-terminal peptide, while C-terminal peptide of type I collagen can be degraded to produce C-terminal telopeptide (23). In this study, osteoclast-related factor CTSK was up-regulated after GC intervention. After 6 weeks of intervention, CTSK mRNA expression was down-regulated. This indicates that Gumi Bao decoction could be used to regulate DKK1 and inhibit osteoclasts in order to prevent and treat GIOP.

\section{Conclusions}

The results of this study show that vertebral DKK1 mRNA was significantly up-regulated, osteoblastic regulator Runx2 mRNA was significantly down-regulated, and osteoclast regulator CTSK mRNA was significantly up-regulated after intervention in the Met group. Our findings indicate that the imbalance of bone homeostasis in GIOP is caused by the influence of increased bone resorption and decreased osteogenesis. After intervention, in the GBDM group and the GBDH group, the mRNA expression of DKK1 and CTSK was down-regulated and that of Runx2 was up- regulated, which suggests that Gumi Bao decoction can regulate DKK1 to promote osteogenesis, inhibit osteoclast, and regulate bone metabolism via the $\mathrm{Wnt} / \beta$-catenin signaling pathway to prevent and treat GIOP.

\section{Acknowledgments}

Funding: This work is supported by the Taicang Science and Technology Bureau Key Research and Development Plan (Social Development) (TC2017SFYL10).

\section{Footnote}

Reporting Checklist: The authors have completed the ARRIVE reporting checklist. Available at http://dx.doi. org/10.21037/apm-20-1340

Data Sharing Statement: Available at http://dx.doi. org/10.21037/apm-20-1340

Conflicts of Interest: All authors have completed the ICMJE uniform disclosure form (available at http://dx.doi. org/10.21037/apm-20-1340). The authors have no conflicts of interest to declare.

Ethical Statement: The authors are accountable for all aspects of the work in ensuring that questions related to the accuracy or integrity of any part of the work are appropriately investigated and resolved. Experiments were performed under a project license (ID: 201901) granted by the Animal Care Welfare Committee of Taicang TCM Hospital Affiliated to Nanjing University of Chinese Medicine, in compliance with Chinese national guidelines for the care and use of animals.

Open Access Statement: This is an Open Access article distributed in accordance with the Creative Commons Attribution-NonCommercial-NoDerivs 4.0 International License (CC BY-NC-ND 4.0), which permits the noncommercial replication and distribution of the article with the strict proviso that no changes or edits are made and the original work is properly cited (including links to both the formal publication through the relevant DOI and the license). See: https://creativecommons.org/licenses/by-nc-nd/4.0/.

\section{References}

1. Whittier X, Saag KG. Glucocorticoid-induced 
osteoporosis. Rheum Dis Clin North Am 2016;42:177-89.

2. Maricic M, Deal C, Dore R, et al. American college of rheumatology guideline for the prevention and treatment of glucocorticoid-induced osteoporosis: comment on the article by Buckley et al. Arthritis Care Res (Hoboken) 2018;70:949-50.

3. Güler-Yüksel M, Hoes JN, Bultink IEM, et al. Glucocorticoids, inflammation and bone. Calcif Tissue Int 2018;102:592-606.

4. Ciccarelli F, De Martinis M, Ginaldi L. Glucocorticoids in patients with rheumatic disease: friends or enemies of bone? Curr Med Chem 2015;22:596-603.

5. Balasubramanian A, Wade SW, Adler RA, et al. Glucocorticoid exposure and fracture risk in patients with new-onset rheumatoid arthritis. Osteoporos Int 2016;27:3239-49.

6. van Staa TP, Leufkens HG, Abenhaim L, et al. Oral corticosteroids and fracture risk: relationship to daily and cumulative doses. Rheumatology (Oxford) 2000;39:1383-9.

7. Adami G, Saag KG. Glucocorticoid-induced osteoporosis: 2019 concise clinical review. Osteoporos Int 2019;30:1145-56.

8. Jing Y, Wang X, Yu J, et al. Associations of serum sex hormone binding globulin with bone mineral densities and higher 10-year probability of fractures in postmenopausal women with type 2 diabetes mellitus. Ann Transl Med 2019;7:457.

9. Canalis E, Mazziotti G, Giustina A, et al. Glucocorticoidinduced osteoporosis: pathophysiology and therapy. Osteoporos Int 2007;18:1319-28.

10. Krasselt M, Baerwald C. An update on glucocorticoidinduced osteoporosis. Dtsch Med Wochenschr 2016;141:352-7.

11. Huang R, Li X, Xu S, et al. Acupoint injection treatment for primary osteoporosis: a systematic review and metaanalysis of randomized controlled trials. Ann Palliat Med 2019;8:586-95.

12. Melton LJ, Thamer M, Ray NF, et al. Fracture attributable to osteoporosis: report from the National Osteoporosis Foundation, J Bone Miner Res 1997;12:16-23.

13. Zhang Q, Cai W, Wang G, et al. Prevalence and contributing factors of osteoporosis in the elderly over 70 years old: an epidemiological study of several community health centers in Shanghai. Ann Palliat Med 2020;9:231-8.

14. Westendorf JJ, Kahler RA, Schroeder TM. Wnt signaling in osteoblasts and bone diseases. GENE 2004;341:19-39.

15. Wang Y, Li YP, Paulson C, et al. Wnt and the Wnt signaling pathway in bone development and disease. Front Biosci (Landmark Ed) 2014;19:379.

16. Ke HZ, Richards EG, Li X, et al. Sclerostin and Dickkopf-1 as Therapeutic Targets in Bone Diseases. Endocr Rev 2012;33:747-83.

17. Xingbo W, Pengbiao L, Shifang G, et al. CircRNA_0006393 promotes osetogensis in glucocorticoid-induced osteoporosis by sponging miR145-5p and upregulating FOXO1. Mol Med Rep 2019;20:2851-8.

18. Kim MS, Day CJ, Selinger CI, et al. MCP-1-induced human osteoclast-like cells are tartrate-resistant acid phosphatase, NFATc1 and calcitonin receptor-positive but require receptor activator of NFkappaB ligand for bone resorption. J Biol Chem 2006;281:1274-85.

19. Canalis E, Economides AN, Gazzerro E. Bone morphogenetic proteins, their antagonists, and the skeleton. Endocr Rev 2003;24:218-35.

20. MacDonald BT, Joiner DM, Oyseman SM, et al. Bone mass is inversely proportional to DKK1 levels in mice. Bone 2007;41:331-9.

21. Ma Y, Yang H, Huang J. Icariin ameliorates dexamethasone induced bone deterioration in an experimental mouse model via activation of microRNA186 inhibition of cathepsin K. Mol Med Rep 2018;17:1633-41.

22. Komori T. Requisite roles of Runx 2 and Cbfb in skeletal development. J Bone Miner Metab 2003;21:193-7.

23. Garnero P. New developments in biological markers of bone metabolism in osteoporosis. Bone 2014;66:46-55.
Cite this article as: Feng $\mathrm{H}$, Gong $\mathrm{Z}$, Liang $\mathrm{G}$, Shen $\mathrm{X}$, Cheng J, Su F, Yang L, Liu H. Gumi Bao decoction regulates bone metabolism-related mRNA expression in glucocorticoidinduced osteoporosis in rats. Ann Palliat Med 2020;9(5):3213-3221. doi: 10.21037/apm-20-1340 